\title{
DETERMINANTS OF RANDOM MATRICES AND JACK POLYNOMIALS OF RECTANGULAR SHAPE
}

\author{
G.E. ANDREWS, I.P. GOULDEN, AND D.M. JACKSON
}

\begin{abstract}
We consider an $N$-dimensional real integral, indexed by a parameter that specifies the power of a Vandermonde determinant. For two particular values of the parameter, this integral arises from matrix integrals, over real symmetric and complex Hermitian $N \times N$ matrices. When it is normalized, it gives the expectation of an arbitrary power of the determinant. The results are given as finite summations, using terminating hypergeometric series. We relate the integral to a specific coefficient in the Jack polynomial indexed by a partition of rectangular shape, and present data for this coefficient in terms of the parameter $\alpha$.
\end{abstract}

\section{INTRODUCTION}

Let $\mathbf{x}=\left(x_{1}, \ldots, x_{N}\right)$ and $V(\mathbf{x})=\prod_{1 \leq i<j \leq N}\left(x_{j}-x_{i}\right)$, and let $\langle\cdot\rangle_{N, \alpha}$ be the normalized expectation operator defined by

$$
\langle f(\mathbf{x})\rangle_{N, \alpha}=\frac{\int_{\mathbb{R}^{N}}|V(\mathbf{x})|^{2 / \alpha} e^{-p_{2}(\mathbf{x}) / 2 \alpha} f(\mathbf{x}) d \mathbf{x}}{\int_{\mathbb{R}^{N}}|V(\mathbf{x})|^{2 / \alpha} e^{-p_{2}(\mathbf{x}) / 2 \alpha} d \mathbf{x}},
$$

where $p_{2}(\mathbf{x})=x_{1}^{2}+\cdots+x_{N}^{2}$, the power sum in $\mathbf{x}$ of degree 2 . A result conjectured by Goulden and Jackson [GJ1] and proved by Okounkov [O] states that

$$
\left\langle J_{\theta}(\mathbf{x} ; \alpha)\right\rangle_{N, \alpha}=J_{\theta}\left(\mathbf{1}_{N} ; \alpha\right)\left(\left[p_{2}^{m}\right] J_{\theta}\right),
$$

where $\mathbf{1}_{N}$ is the vector with $N$ ones and $\theta$ is a partition of $2 m, J_{\theta}$ is the Jack symmetric function indexed by $\theta$, and $[A] B$ denotes the coefficient of $A$ in the expansion of $B$. By determining $\left\langle J_{\theta}(\mathbf{x} ; \alpha)\right\rangle_{N, \alpha}$ we will thus obtain explicit expressions for $\left[p_{2}^{m}\right] J_{\theta}$ for particular cases of $\theta$ and $\alpha$.

The purpose of this paper is to examine this question in detail in the cases $\alpha=1,2$. We regard the details of integrating $f(\mathbf{x})=\left(x_{1} \cdots x_{N}\right)^{u}$ with $\alpha=2$ as complementary to the integration carried out in [GJ1], in which $f(\mathbf{x})=x_{1}^{k}$, giving the generating series for monopoles in nonorientable surfaces. A monopole is a graph with a single vertex, and the generating series for monopoles in orientable surfaces was obtained through a matrix integral in [HZ]. We also hope that these integrations will provide clues to this integration for an arbitrary $\alpha$, as was carried out in [GHJ], where $\alpha$ appeared in a parameterization for virtual Euler characteristics of the moduli spaces of real and complex algebraic curves.

Date: May 28, 2002.

1991 Mathematics Subject Classification. Primary 15A52; Secondary 05E05.

Key words and phrases. Jack polynomials, matrix integrals. 
For a non-negative integer $u$, and a positive real number $a$, the constituent of (1.1) we consider in detail is

$$
\mathcal{I}_{N, \alpha}^{(u, a)}=\int_{\mathbb{R}^{N}}|V(\mathbf{x})|^{2 / \alpha} e^{-a p_{2}(\mathbf{x})}\left(x_{1} \cdots x_{N}\right)^{u} d \mathbf{x},
$$

and from this we determine the normalized expectation of $\left(x_{1} \cdots x_{N}\right)^{u}$ by

$$
\left\langle\left(x_{1} \cdots x_{N}\right)^{u}\right\rangle_{N, \alpha}=\mathcal{I}_{N, \alpha}^{\left(u, \frac{1}{2 \alpha}\right)} / \mathcal{I}_{N, \alpha}^{\left(0, \frac{1}{2 \alpha}\right)}
$$

The main results of this paper give the evaluation of (1.3), and are stated in Theorem 2.1 for $\alpha=1$, and in Theorems 5.1 and 6.1 for $\alpha=2$.

In [DC], expressions for the normalized expectation (1.4) are also obtained when $\alpha=1,2$. In the present paper the results and their derivations are simpler than those given in $[\mathrm{DC}]$. As described in [DC], these normalized expectations give the moments of the determinant distribution for Hermitian $(\alpha=1)$ and real symmetric matrices $(\alpha=2)$.

\section{THE CASE $\alpha=1$}

Throughout, we use the following fundamental property of the Vandermonde determinant:

$$
V(\mathbf{x})=\operatorname{det}\left[x_{j}^{i-1}\right]_{N \times N}=\operatorname{det}\left[P_{i-1}\left(x_{j}\right)\right]_{N \times N},
$$

where $P_{i-1}(x)$ is any monic polynomial of degree $i-1$, and the determinants are indexed with $i, j \in\{1, \ldots, N\}$. (We index all determinants in this paper with $i, j \geq 1$.)

We now consider the evaluation of $\mathcal{I}_{N, 1}^{(u, a)}$, and begin by scaling the variables, to obtain

$$
\mathcal{I}_{N, 1}^{(u, a)}=(2 a)^{-N(u+N) / 2} \mathcal{I}_{N, 1}^{\left(u, \frac{1}{2}\right)}
$$

Let $\mathcal{H}_{n}(x)$ for $n \geq 0$ denote the monic Hermite polynomial of degree $n$ in $x$ (see, e.g., [C], p. 133), with generating series $\sum_{n \geq 0} \mathcal{H}_{n}(x) w^{n} / n !=e^{x w-w^{2} / 2}$. Thus, from (2.1) we have

$$
|V(\mathbf{x})|^{2}=V(\mathbf{x})^{2}=\operatorname{det}\left[x_{j}^{i-1}\right]_{N \times N} \operatorname{det}\left[\mathcal{H}_{i-1}\left(x_{j}\right)\right]_{N \times N},
$$

so, from (1.3) we obtain

$$
\begin{aligned}
\mathcal{I}_{N, 1}^{\left(u, \frac{1}{2}\right)} & =\int_{\mathbb{R}^{N}} \operatorname{det}\left[x_{j}^{i+u-1}\right]_{N \times N} \operatorname{det}\left[\mathcal{H}_{i-1}\left(x_{j}\right)\right]_{N \times N} e^{-p_{2}(\mathbf{x}) / 2} d \mathbf{x} \\
& =\sum_{\pi \in \mathfrak{S}_{N}} \operatorname{sgn}(\pi) \operatorname{det}\left[\int_{\mathbb{R}} x^{i+u-1} \mathcal{H}_{\pi(j)-1}(x) e^{-x^{2} / 2} d x\right]_{N \times N} \\
& =N ! \operatorname{det}\left[\int_{\mathbb{R}} x^{i+u-1} \mathcal{H}_{j-1}(x) e^{-x^{2} / 2} d x\right]_{N \times N} .
\end{aligned}
$$

But from the generating series for the Hermite polynomials above, it follows immediately that

$$
\int_{\mathbb{R}} x^{k} \mathcal{H}_{n}(x) e^{-x^{2} / 2} d x= \begin{cases}\sqrt{\pi} k ! 2^{\frac{1}{2}(n-k+1)} \frac{1}{\left(\frac{1}{2}(k-n)\right) !} & \text { if } k \equiv n \quad(\bmod 2) \text { and } k \geq n \\ 0 & \text { otherwise }\end{cases}
$$


Then

$$
\mathcal{I}_{N, 1}^{\left(u, \frac{1}{2}\right)}=\sqrt{\pi}^{N} \frac{N !}{2^{\frac{N}{2}(u-1)}}\left(\prod_{i=1}^{N}(i+u-1) !\right) D_{N, u}
$$

where

$$
D_{N, u}=\operatorname{det}\left[\frac{1}{\left(\frac{1}{2}(i-j+u)\right) !}\right]_{N \times N}
$$

with the convention that $\left(\frac{1}{2}(i-j+u)\right)$ ! is to be replaced by 0 if $i-j+u$ is odd. There are two cases.

Case 1: Suppose $u$ is even. Let $u=2 t$ and $\Delta_{m, t}=\operatorname{det}\left[(i-j+t) !^{-1}\right]_{m \times m}$. Then, by row and column permutations that bring this matrix to a block diagonal form, $D_{N, u}=\Delta_{m, t}^{2}$ if $N=2 m$ and $D_{N, u}=\Delta_{m, t} \Delta_{m+1, t}$ if $N=2 m+1$. Now

$$
\Delta_{m, t}=\left(\prod_{i=1}^{m} \frac{1}{(i-1+t) !}\right) \operatorname{det}\left[(i-1+t)_{j-1}\right]_{m \times m},
$$

where $(x)_{n}=x(x-1) \cdots(x-n+1)$ is the falling factorial. But $(x)_{j-1}$ is a monic polynomial of degree $j-1$ in $x$, so from (2.1) we obtain

$$
\operatorname{det}\left[(i-1+t)_{j-1}\right]_{m \times m}=\prod_{1 \leq i<j \leq m}(j-1+t-(i-1+t))=\prod_{i=1}^{m}(i-1) !
$$

and thus we have

$$
\Delta_{m, t}=\prod_{i=1}^{m} \frac{(i-1) !}{(i-1+t) !} .
$$

Case 2: Suppose $u$ is odd, with $u=2 t+1$. Then, by row and column permutations that bring this matrix to a block diagonal form, $D_{N, u}=(-1)^{m} \Delta_{m, t+1} \Delta_{m, t}$ if $N=2 m$ and $D_{N, u}=0$ if $N=2 m+1$.

Combining the two cases we obtain the following result.

Theorem 2.1. Let

$$
d_{N, u}=\frac{\sqrt{\pi}^{N} N !}{2^{\frac{N}{2}(u-1)}} \prod_{i=1}^{N}(i+u-1) !,
$$

and $\Delta_{m, t}$ be given by (2.4).Then

$$
\mathcal{I}_{N, 1}^{\left(u, \frac{1}{2}\right)}= \begin{cases}d_{N, u} \Delta_{m, t}^{2} & \text { if } N=2 m, u=2 t \\ d_{N, u} \Delta_{m, t} \Delta_{m+1, t} & \text { if } N=2 m+1, u=2 t \\ d_{N, u}(-1)^{m} \Delta_{m, t+1} \Delta_{m, t} & \text { if } N=2 m, u=2 t+1, \\ 0 & \text { if } N=2 m+1, u=2 t+1 .\end{cases}
$$

The corresponding normalizations are found, from (1.4), to be

$$
\left\langle\left(x_{1} \cdots x_{N}\right)^{u}\right\rangle_{N, 1}= \begin{cases}e_{N, u} \Delta_{m, t}^{2} & \text { if } N=2 m, u=2 t \\ e_{N, u} \Delta_{m, t} \Delta_{m+1, t} & \text { if } N=2 m+1, u=2 t \\ e_{N, u}(-1)^{m} \Delta_{m, t+1} \Delta_{m, t} & \text { if } N=2 m, u=2 t+1 \\ 0 & \text { if } N=2 m+1, u=2 t+1\end{cases}
$$


where

$$
e_{N, u}=2^{-\frac{N u}{2}} \prod_{i=1}^{N} \frac{(i+u-1) !}{(i-1) !}
$$

\section{The CASE $\alpha=2$}

We now consider the evaluation of $\mathcal{I}_{N, 2}^{(u, a)}$, and begin by scaling the variables, to obtain

$$
\mathcal{I}_{N, 2}^{(u, a)}=(2 a)^{-N(2 u+N+1) / 4} \mathcal{I}_{N, 2}^{\left(u, \frac{1}{2}\right)} .
$$

Let $\psi_{i}(x)=x^{i} e^{-x^{2} / 2}$, and $\Psi_{i}(x)=\int_{-\infty}^{x} \psi_{i}(t) d t$. Let

$$
\langle f, g\rangle=\frac{1}{2} \int_{-\infty<y<x<\infty}^{(f(y) g(x)-f(x) g(y)) d x d y .}
$$

Now clearly $\langle f, g\rangle=-\langle g, f\rangle$ when both are defined, so $\langle\cdot, \cdot\rangle$ is a skew-symmetric bilinear form. The other properties of this bilinear form that are of interest arise when $f, g$ are even or odd functions.

If $f, g$ are both even or both odd, then substituting $x=-y, y=-x$ in the integral gives $\langle f, g\rangle=-\langle f, g\rangle$, so we conclude that $\langle f, g\rangle=0$ in this case. If one of $f, g$ is even and the other is odd, then the same substitution gives

$$
\int_{-\infty<y<x<\infty} f(y) g(x) d x d y=-\int_{-\infty<y<x<\infty}^{f(x) g(y) d x d y}
$$

and we conclude in this case that

$$
\langle f, g\rangle=\int_{-\infty<y<x<\infty}^{f(y) g(x) d x} d y
$$

Now for $\mathbf{x}$ in the principal cone $\mathcal{C}=\left\{\mathbf{x}:-\infty<x_{1}<\ldots<x_{N}<\infty\right\}$, we have $|V(\mathbf{x})|=V(\mathbf{x})$, so from (1.3) and (2.1), by symmetry we have

$$
\mathcal{I}_{N, 2}^{\left(u, \frac{1}{2}\right)}=N ! \int_{\mathcal{C}} \operatorname{det}\left[\psi_{i+u-1}\left(x_{j}\right)\right]_{N \times N} d \mathbf{x}
$$

We treat the even and odd cases of $N$ separately, continuing now with the even case, but deferring the odd case to Section 6 .

Let $N=2 n$, where $\mathrm{n}$ is a positive integer. Integrating over variables with odd index in (3.3), with the convention that $x_{0}=-\infty$, we have

$$
\begin{aligned}
\mathcal{I}_{N, 2}^{\left(u, \frac{1}{2}\right)} & =N ! \int_{\mathcal{C}} \operatorname{det}\left[\int_{x_{2 j-2}}^{x_{2 j}} \psi_{i+u-1}\left(x_{2 j-1}\right) d x_{2 j-1}, \psi_{i+u-1}\left(x_{2 j}\right) d x_{2 j}\right]_{N \times N} \\
& =N ! \int_{\mathcal{C}} \operatorname{det}\left[\Psi_{i+u-1}\left(x_{2 j}\right)-\Psi_{i+u-1}\left(x_{2 j-2}\right), \psi_{i+u-1}\left(x_{2 j}\right) d x_{2 j}\right]_{N \times N},
\end{aligned}
$$

where the elements of the matrix are indexed by $i=1, \ldots, N$ and $j=1, \ldots, n$ (so the matrix is $N \times N)$. Now $\Psi_{i+u-1}\left(x_{0}\right)=0$ for all $i$, so the determinant in the integral above is equal to

$$
\operatorname{det}\left[\Psi_{i+u-1}\left(x_{2 j}\right), \psi_{i+u-1}\left(x_{2 j}\right) d x_{2 j}\right]_{N \times N}
$$


by elementary column operations on the columns with odd index. Then, on resymmetrizing,

$$
\mathcal{I}_{N, 2}^{\left(u, \frac{1}{2}\right)}=\frac{N !}{n !} \int_{\mathbb{R}^{n}} \operatorname{det}\left[\Psi_{i+u-1}\left(x_{2 j}\right), \psi_{i+u-1}\left(x_{2 j}\right) d x_{2 j}\right]_{N \times N} .
$$

But the corresponding integral of the $2 \times 2$ subdeterminant with columns $2 j-1,2 j$ and rows $i, m$ leads to

$$
\int_{-\infty}^{\infty} \operatorname{det}\left[\begin{array}{ll}
\Psi_{i+u-1}\left(x_{2 j}\right) & \psi_{m+u-1}\left(x_{2 j}\right) d x_{2 j} \\
\Psi_{m+u-1}\left(x_{2 j}\right) & \psi_{i+u-1}\left(x_{2 j}\right) d x_{2 j}
\end{array}\right]=2\left\langle\psi_{i+u-1}, \psi_{m+u-1}\right\rangle,
$$

which is equal to 0 if $i, m$ have the same parity (since $\psi_{k}$ is an even or odd function when $k$ is even or odd, respectively). Thus, expanding the determinant iteratively by Laplace's expansion with column partition $(\{1,2\}, \ldots,\{N-1, N\})$, and evaluating the $2 \times 2$ subdeterminants by means of (3.5), we have

$$
\begin{aligned}
\mathcal{I}_{N, 2}^{\left(u, \frac{1}{2}\right)} & =\frac{N !}{n !} \sum_{\sigma, \pi \in \mathfrak{S}_{n}} \operatorname{sgn}(\sigma) \operatorname{sgn}(\pi) \prod_{j=1}^{n} 2\left\langle\psi_{2 \sigma(j)-1+u-1}, \psi_{2 \pi(j)+u-1}\right\rangle \\
& =2^{n} N ! \sum_{\omega \in \mathfrak{S}_{n}} \operatorname{sgn}(\omega) \prod_{j=1}^{n}\left\langle\psi_{2 \omega(j)+u-2}, \psi_{2 j+u-1}\right\rangle,
\end{aligned}
$$

where we have set $\omega=\sigma \pi^{-1}$. Here $\sigma$ describes the action of the column permutation for odd indices, by sending column $2 j-1$ to row $2 \sigma(j)-1$, and $\pi$ describes the action for even indices, by sending column $2 j$ to row $2 \pi(j)$, and there are no other non-zero terms in the expansion of the determinant. Finally, for $N=2 n$, we have

$$
\mathcal{I}_{N, 2}^{\left(u, \frac{1}{2}\right)}=2^{n} N ! \operatorname{det}\left[\left\langle\psi_{2 i+u-2}, \psi_{2 j+u-1}\right\rangle\right]_{n \times n} .
$$

$$
\text { 4. Evaluation of }\left\langle\psi_{2 i+u-2}, \psi_{2 j+u-1}\right\rangle
$$

We now evaluate $\left\langle\psi_{2 i+u-2}, \psi_{2 j+u-1}\right\rangle$, the entry in the determinant (3.6). Let $(x)^{(n)}=x(x+1) \cdots(x+n-1)$, the rising factorial.

Lemma 4.1. Let $i, j$ be positive integers, $u$ a non-negative integer, and

$$
c(i, j, u)=(-1)^{u} \sqrt{\pi}\left(\frac{1}{2}\right)^{(i+j+u-2)}{ }_{2} F_{1}\left[\begin{array}{cc}
1, & -i+1-\left\lfloor\frac{u}{2}\right\rfloor \\
-, & \frac{5}{2}-i-j-u
\end{array} \mid 2\right],
$$

a finite summation. Then, for $u$ odd,

$$
\left\langle\psi_{2 i+u-2}, \psi_{2 j+u-1}\right\rangle=c(i, j, u)
$$

and, for u even,

$$
\left\langle\psi_{2 i+u-2}, \psi_{2 j+u-1}\right\rangle=c(j, i, u) .
$$

Proof. Let $K \geq 1$ and $M \geq 0$ be integers of opposite parity. Then, from (3.2), and integrating by parts with respect to $y$ we have the recursion

$$
\left\langle\psi_{K}, \psi_{M}\right\rangle=-\sqrt{\pi}\left(\frac{1}{2}\right)^{\left(\frac{K+M-1}{2}\right)}+(K-1)\left\langle\psi_{K-2}, \psi_{M}\right\rangle .
$$

Let $m, k$ be non-negative integers. Substitute $K=2 k+1, M=2 m$ in (4.1), and iterate, to obtain

$$
\left\langle\psi_{2 k+1}, \psi_{2 m}\right\rangle=-\sqrt{\pi}\left(\frac{1}{2}\right)^{(m+k)}{ }_{2} F_{1}\left[\begin{array}{cc|}
1, & -k \\
-, & \frac{1}{2}-m-k
\end{array} \mid 2\right] .
$$


The result follows immediately for $u$ odd, by substitution of $k=i+\frac{u-3}{2}$ and $m=j+\frac{u-1}{2}$ in (4.2). For $u$ even, apply the skew-symmetry of the bilinear form and the result follows by substitution of $k=j-1+\frac{u}{2}$ and $m=i-1+\frac{u}{2}$ in (4.2).

\section{Evaluation of the Determinant}

We now return to the evaluation of the determinant in (3.6). Replacing $K$ by $2 i+u$ and $M$ by $2 j+u-1$ in (4.1), and rewriting, we obtain

$$
\left\langle\psi_{2 i+u-2}, \psi_{2 j+u-1}\right\rangle-\frac{1}{2 i+u-1}\left\langle\psi_{2 i+u}, \psi_{2 j+u-1}\right\rangle=\sqrt{\pi} \frac{\left(\frac{1}{2}\right)^{(i+j+u-1)}}{2 i+u-1},
$$

and applying this sequentially as a row operation, starting with row 1 of the determinant in (3.6), we have

$$
\begin{aligned}
\operatorname{det}\left[\left\langle\psi_{2 i+u-2}, \psi_{2 j+u-1}\right\rangle\right]_{n \times n} & =\frac{\sqrt{\pi}^{n-1}}{\prod_{i=1}^{n-1}(2 i+u-1)} \operatorname{det}\left[\begin{array}{l}
\left.\left[\left(\frac{1}{2}\right)^{(i+j+u-1)}\right]\right]_{(n-1) \times n} \\
{\left[\left\langle\psi_{2 n+u-2}, \psi_{2 j+u-1}\right\rangle\right]_{1 \times n}}
\end{array}\right]_{n \times n} \\
& =\sqrt{\pi}^{n-1}\left(\prod_{i=1}^{n-1} \frac{\left(\frac{1}{2}\right)^{(i+u)}}{2 i+u-1}\right) \operatorname{det}\left[\begin{array}{l}
{\left[\left(i+u+\frac{1}{2}\right)^{(j-1)}\right]_{(n-1) \times n}} \\
{\left[\left\langle\psi_{2 n+u-2}, \psi_{2 j+u-1}\right\rangle\right]_{1 \times n}}
\end{array}\right]_{n \times n} .
\end{aligned}
$$

Now multiply the $j+1$ st column of this determinant by $a_{j}$, where

$$
a_{j}=\frac{(-n+1)^{(j)}}{j !\left(\frac{3}{2}+u\right)^{(j)}}, \quad j=0, \ldots n-1,
$$

sum over $j$ from 0 to $n-1$, and replace column $n$ by this sum. The $i$ th row of the new column $n$ is therefore

$$
\begin{aligned}
\sum_{j=0}^{n-1} \frac{(-n+1)^{(j)}\left(i+u+\frac{1}{2}\right)^{(j)}}{j !\left(\frac{3}{2}+u\right)^{(j)}} & ={ }_{2} F_{1}\left[\begin{array}{cc}
i+u+\frac{1}{2}, & 1-n \\
-, & \frac{3}{2}+u
\end{array}\right] \\
& =\frac{(-i+1)^{(n-1)}}{\left(\frac{3}{2}+u\right)^{(n-1)}}=0, \quad i=1, \ldots n-1,
\end{aligned}
$$

where we have applied Vandermonde's summation to evaluate the terminating hypergeometric series. Thus we have

$$
\operatorname{det}\left[\left\langle\psi_{2 i+u-2}, \psi_{2 j+u-1}\right\rangle\right]_{n \times n}=\frac{\sqrt{\pi}^{n-1}}{a_{n-1}}\left(\prod_{i=1}^{n-1} \frac{\left(\frac{1}{2}\right)^{(i+u)}}{2 i+u-1}\right) T_{n-1} A_{n},
$$

where $T_{n-1}=\operatorname{det}\left[\left(i+u+\frac{1}{2}\right)^{(j-1)}\right]_{(n-1) \times(n-1)}$, and

$$
A_{n}=\sum_{j=0}^{n-1} a_{j}\left\langle\psi_{2 n+u-2}, \psi_{2 j+u+1}\right\rangle
$$

Now $(x)^{(j-1)}$ is a monic polynomial of degree $j-1$ in $x$, so from $(2.1)$ we obtain

$$
T_{n-1}=\prod_{0 \leq i<j \leq n-2}\left(j+u+\frac{1}{2}-\left(i+u+\frac{1}{2}\right)\right)=\prod_{i=0}^{n-2} i \text { ! }
$$


For $A_{n}$, we apply Lemma 4.1 with $i=n$ and $j$ replaced by $j+1$. There are two cases; for $u$ odd, we obtain

$$
A_{n}=-\sqrt{\pi} \sum_{j=0}^{n-1} \frac{(-n+1)^{(j)}\left(\frac{1}{2}\right)^{(n+j+u-1)}}{j !\left(\frac{3}{2}+u\right)^{(j)}} \sum_{l \geq 0} \frac{\left(\frac{3}{2}-n-\frac{u}{2}\right)^{(l)} 2^{l}}{\left(\frac{3}{2}-n-j-u\right)^{(l)}} .
$$

Then, by reversing the order of these finite summations and rearranging,

$$
A_{n}=-\sqrt{\pi}\left(\frac{1}{2}\right)^{(n+u-1)} \sum_{l \geq 0} \frac{\left(\frac{3}{2}-n-\frac{u}{2}\right)^{(l)} 2^{l}}{\left(\frac{3}{2}-n-u\right)^{(l)}}{ }_{2} F_{1}\left[\begin{array}{cc}
-n+1, & -\frac{1}{2}+n+u-l \\
-, & \frac{3}{2}+u
\end{array} \mid 1\right] \text {. }
$$

By Vandermonde's summation, and excluding the initial values of $l$ corresponding to terms that give zero contribution, we obtain

$$
A_{n}=-\sqrt{\pi}\left(\frac{1}{2}\right)^{(n+u-1)} \sum_{l \geq n-1} \frac{\left(\frac{3}{2}-n-\frac{u}{2}\right)^{(l)} 2^{l}}{\left(\frac{3}{2}-n-u\right)^{(l)}} \frac{(2-n+l)^{(n-1)}}{\left(\frac{3}{2}+u\right)^{(n-1)}},
$$

whence, by shifting the summation range to 0 and rearranging,

$$
A_{n}=-\sqrt{\pi} 2^{n-1}(n-1) ! \frac{\left(\frac{u+1}{2}\right)^{(n-1)}\left(\frac{1}{2}\right)^{(u)}}{\left(u+\frac{3}{2}\right)^{(n-1)}}{ }_{2} F_{1}\left[\begin{array}{cc}
n, & \frac{1-u}{2} \\
-, & \frac{1}{2}-u
\end{array} \mid 2\right],
$$

in the case that $u$ is odd.

In the other case, for $u$ even, we obtain

$$
A_{n}=\sqrt{\pi} \sum_{j=0}^{n-1} \frac{(-n+1)^{(j)}\left(\frac{1}{2}\right)^{(n+j+u-1)}}{j !\left(\frac{3}{2}+u\right)^{(j)}} \sum_{l \geq 0} \frac{\left(-j-\frac{u}{2}\right)^{(l)} 2^{l}}{\left(\frac{3}{2}-n-j-u\right)^{(l)}} .
$$

Then, by reversing the order of these finite summations and rearranging,

$$
A_{n}=\sqrt{\pi}\left(\frac{1}{2}\right)^{(n+u-1)} \sum_{l \geq 0} \frac{\left(-\frac{u}{2}\right)^{(l)} 2^{l}}{\left(\frac{3}{2}-n-u\right)^{(l)}}{ }_{3} F_{2}\left[\begin{array}{ccc}
-n+1, & -\frac{1}{2}+n+u-l, & 1+\frac{u}{2} \\
-, & \frac{3}{2}+u, & 1+\frac{u}{2}-l
\end{array} \mid 1\right] \text {. }
$$

By Saalschutz's summation, and excluding the initial values of $l$ corresponding to terms that give zero contribution, we obtain

$$
A_{n}=\sqrt{\pi}\left(\frac{1}{2}\right)^{(n+u-1)} \sum_{l \geq n-1} \frac{\left(-\frac{u}{2}\right)^{(l)} 2^{l}}{\left(\frac{3}{2}-n-u\right)^{(l)}} \frac{(-l)^{(n-1)}\left(\frac{3}{2}-\frac{u}{2}-n\right)^{(n-1)}}{\left(1+\frac{u}{2}-l\right)^{(n-1)}\left(\frac{1}{2}-n-u\right)^{(n-1)}},
$$

whence, by shifting the summation range to 0 and rearranging,

$$
A_{n}=\sqrt{\pi}(-2)^{n-1}(n-1) ! \frac{\left(\frac{u+1}{2}\right)^{(n-1)}\left(\frac{1}{2}\right)^{(u)}}{\left(u+\frac{3}{2}\right)^{(n-1)}}{ }_{2} F_{1}\left[\begin{array}{cc}
n, & \frac{-u}{2} \\
-, & \frac{1}{2}-u
\end{array} \mid 2\right],
$$

in the case that $u$ is even.

Theorem 5.1. Let $N=2 n$, where $n$ is a positive integer. Then

$$
\mathcal{I}_{N, 2}^{\left(u, \frac{1}{2}\right)}=(-1)^{n u}(2 \sqrt{\pi})^{n} N !\left(\prod_{j=0}^{n-1} j !\left(\frac{1}{2}\right)^{(j+u)}\right){ }_{2} F_{1}\left[\begin{array}{cc}
n, & -\left\lfloor\frac{u}{2}\right\rfloor \\
-, & \frac{1}{2}-u
\end{array} \mid 2\right],
$$

a finite summation.

Proof. This follows directly from (3.6), (5.2), (5.3), (5.4) and (5.5). 
The corresponding normalization for $N=2 n$ is found, from (1.4) and (3.1) with $a=\frac{1}{4}$, to be

$$
\left\langle\left(x_{1} \cdots x_{N}\right)^{u}\right\rangle_{N, 2}=(-1)^{n u} u !\left(\prod_{j=1}^{u} \frac{(2 n+2 j-3) ! !}{(2 j-1) ! !}\right) \sum_{k=0}^{\left\lfloor\frac{u}{2}\right\rfloor}\left(\begin{array}{c}
-n \\
k
\end{array}\right)\left(\begin{array}{c}
u-\frac{1}{2}-k \\
\left\lfloor\frac{u}{2}\right\rfloor-k
\end{array}\right) 2^{k},
$$

where we have written the hypergeometric function as an explicit summation.

\section{The CASE N ODD}

We now return to the odd case of $N$ in (3.3). Let $N=2 n+1$, where $n$ is a nonnegative integer. The approach is very similar to the even case. By integrating over variables with odd index, applying elementary column operations to the columns with odd index, and resymmetrizing, we obtain in this case (c.f. expression (3.4) for the even case)

$$
\mathcal{I}_{N, 2}^{\left(u, \frac{1}{2}\right)}=\frac{N !}{n !} \int_{\mathbb{R}^{n}} \operatorname{det}[\underbrace{\left[\Psi_{i+u-1}\left(x_{2 j}\right), \psi_{i+u-1}\left(x_{2 j}\right) d x_{2 j}\right]}_{j=1, \ldots, n}, \Psi_{i+u-1}(\infty)]_{N \times N} .
$$

Then, expanding the determinant iteratively by Laplace's expansion with column partition $(\{1,2\}, \ldots,\{N-2, N-1\},\{N\})$, we obtain in this case

$$
\mathcal{I}_{N, 2}^{\left(u, \frac{1}{2}\right)}=2^{n} N ! \operatorname{det}[\underbrace{\left[\left\langle\psi_{2 i+u-2}, \psi_{2 j+u-1}\right\rangle\right]}_{j=1, \ldots, n}, \Psi_{2 i+u-2}(\infty)]_{(n+1) \times(n+1)} .
$$

To evaluate this determinant, we apply (5.1) sequentially as a row operation, starting with row 1 . For the last column, we have $\Psi_{2 i+u-2}(\infty)-\frac{1}{2 i+u-1} \Psi_{2 i+u}(\infty)=0$, from integrating by parts. Thus

$$
\begin{aligned}
& \mathcal{I}_{N, 2}^{\left(u, \frac{1}{2}\right)}=(2 \sqrt{\pi})^{n} N !\left(\prod_{i=1}^{n} \frac{\left(\frac{1}{2}\right)^{(i+u)}}{2 i+u-1}\right) \operatorname{det}\left[\begin{array}{ll}
{\left[\left(i+u+\frac{1}{2}\right)^{(j-1)}\right]_{n \times n}} & {[0]_{n \times 1}} \\
{\left[\left\langle\psi_{2 n+u}, \psi_{2 j+u-1}\right\rangle\right]_{1 \times n}} & {\left[\Psi_{2 n+u}(\infty)\right]_{1 \times 1}}
\end{array}\right]_{(n+1) \times(n+1)} \\
& =(2 \sqrt{\pi})^{n} N !\left(\prod_{i=1}^{n} \frac{\left(\frac{1}{2}\right)^{(i+u)}}{2 i+u-1}\right) T_{n} \Psi_{2 n+u}(\infty) \text {. }
\end{aligned}
$$

Theorem 6.1. Let $N=2 n+1$, where $n$ is a non-negative integer. Then

$$
\mathcal{I}_{N, 2}^{\left(u, \frac{1}{2}\right)}=\sqrt{\pi}^{n+1} N ! 2^{n+\frac{u+1}{2}}\left(\frac{1}{2}\right)^{\left(\frac{u}{2}\right)} \prod_{j=1}^{n}\left((j-1) !\left(\frac{1}{2}\right)^{(j+u)}\right)
$$

if $u$ is even and is 0 if $u$ is odd.

Proof. Now $\Psi_{2 n+u}(\infty)=\sqrt{\pi} 2^{n+\frac{u+1}{2}}\left(\frac{1}{2}\right)^{\left(n+\frac{u}{2}\right)}$ if $u$ is even and is 0 if $u$ is odd. The result follows from (5.3), after rearrangement.

The corresponding normalization for $N=2 n+1$ is found, from (1.4) and (3.1) with $a=\frac{1}{4}$, to be

$$
\left\langle\left(x_{1} \cdots x_{N}\right)^{u}\right\rangle_{N, 2}=2^{\frac{u}{2}}(u-1) ! ! \prod_{j=1}^{u} \frac{(2 n+2 j-1) ! !}{(2 j-1) ! !}
$$


if $u$ is even and is 0 if $u$ is odd.

Dumitriu [Du] conjectured the form of the normalizations (5.6) and (6.1) for $\alpha=2$, with an unidentified polynomial in $n$ in place of the explicit summation that appears in (5.6).

\section{JACK SYMMETRIC FUNCTIONS}

We may now obtain an expression for the coefficient of $p_{2}^{m}$ in a zonal polynomial or Schur function at a rectangular shape $N \square u=\left[(u)^{N}\right]$, where $m=\frac{N u}{2}$. In view of the possibility of extending this to an expression for the same coefficient in a Jack symmetric function of the same shape, we shall work with Jack symmetric functions and defer specializing the Jack parameter.

Let $\lambda=\left(\lambda_{1}, \lambda_{2}, \ldots\right)$ be a partition and let $\tilde{\lambda}=\left(\tilde{\lambda}_{1}, \tilde{\lambda}_{2}, \ldots\right)$ be the conjugate of $\lambda$. For $w=(i, j) \in \lambda$, let $h(w)=\lambda_{i}+\tilde{\lambda}_{j}-i-j+1$, the hook-length of $w$ in $\lambda$. Let $H_{\lambda}=\prod_{w \in \lambda} h(w)$. Then $J_{\lambda}(\mathbf{x} ; 1)=H_{\lambda} s_{\lambda}(\mathbf{x})$, where $s_{\lambda}$ is a Schur function, and $J_{\lambda}(\mathbf{x} ; 2)=Z_{\lambda}(\mathbf{x})$, where $Z_{\lambda}$ is a zonal polynomial (the normalization is such that $\left[x_{1} \cdots x_{N}\right] J_{\lambda}(\mathbf{x} ; \alpha)=N$ !, where $\lambda$ is a partition of $\left.N\right)$.

We now use (1.2) to obtain an explicit expression for $\left[p_{2}^{m}\right] Z_{N \square u}$. Let $h_{\star}^{\lambda}(i, j)=$ $\tilde{\lambda}_{j}-i+1+\alpha\left(\lambda_{i}-j\right)$, the lower hook-length of $(i, j)$ in $\lambda$, and let

$$
c_{\lambda}(\alpha)=\prod_{i=1}^{l(\lambda)} h_{\star}^{\lambda}(i, 1) .
$$

Then, from Proposition 5.1 of [St],

$$
J_{N \square u}(\mathbf{x} ; \alpha)=\left(\prod_{j=1}^{u} c_{N \square j}(\alpha)\right)\left(x_{1} \cdots x_{N}\right)^{u},
$$

so, from (1.2) we have

$$
\left[p_{2}^{m}\right] J_{N \square u}(\mathbf{x} ; \alpha)=\frac{\prod_{j=1}^{u} c_{N \square j}(\alpha)}{J_{N \square u}\left(\mathbf{1}_{N} ; \alpha\right)}\left\langle\left(x_{1} \cdots x_{N}\right)^{u}\right\rangle_{N, \alpha} .
$$

But Theorem 5.4 of $[\mathrm{St}]$ gives the principal specialization of the Jack symmetric function

$$
J_{N \square u}\left(\mathbf{1}_{N} ; \alpha\right)=\prod_{i=1}^{N} \prod_{j=1}^{u}(N-i+1+\alpha(j-1)),
$$

and Proposition 5.5 of [St] gives $c_{N \square j}(\alpha)=\prod_{i=1}^{N}(N-i+1+\alpha(j-1))$, whence

$$
\left[p_{2}^{m}\right] J_{N \square u}(\mathbf{x} ; \alpha)=\left\langle\left(x_{1} \cdots x_{N}\right)^{u}\right\rangle_{N, \alpha},
$$

which is given explicitly for $\alpha=2$ by (5.6) and (6.1), and for $\alpha=1$ at the end of Section 2.

For $\alpha=1$, as a check on our normalization formulas, note that from the JacobiTrudi formula for Schur functions, we obtain

$$
\left[p_{2}^{m}\right] s_{N \square u}(\mathbf{x})=2^{-\frac{N u}{2}} D_{N, u}
$$

where $D_{N, u}$ is defined in (2.3). Thus

$$
\left[p_{2}^{m}\right] J_{N \square u}(\mathbf{x} ; 1)=2^{-\frac{N u}{2}} H_{N \square u} D_{N, u}
$$


where the hook product $H_{N \square u}$ is found straightforwardly to be

$$
H_{N \square u}=\prod_{i=1}^{N} \frac{(i+u-1) !}{(i-1) !} .
$$

Now observe that

$$
2^{-\frac{N u}{2}} H_{N \square u}=e_{N, u}
$$

where $e_{N, u}$ is defined in (2.5), and we conclude that (7.2) is equivalent to the normalizations given at the end of Section 2, by applying (7.1) with $\alpha=1$.

For $\alpha=2$, we obtain checks on our normalization formulas (5.6) and (6.1) in the Appendix, from the table of coefficients of $p_{2}^{m}$ in Jack functions for rectangular shapes given there.

\section{ACKNOWLEDGEMENTS}

The authors thank Alan Edelman and Richard Stanley for introducing them to this question, and for useful discussions. This work was supported by National Sci-

ence Foundation Grant DMS-9206993 (GEA), and research grants from the Natural Sciences and Engineering Research Council of Canada (IPG and DMJ).

\section{REFERENCES}

[AS] "Handbook of Mathematical Functions," edited by M.Abramowitz and I. Stegun, Dover New York, 1965.

[C] H. Cramer, "Mathematical methods of statistics," Princeton U. Press, Princeton, 1946.

[DC] R. Delannay And G. LE CAËR, Distribution of the determinant of a random real-symmetric matrix from the Gaussian orthogonal ensemble, Physical Review E 62 (2000), 1526-1536.

[Du] I. DumitRIU, private communication, June 1999.

[GHJ] I.P. Goulden, J.L. Harer and D.M. Jackson, A geometric parameterization for the virtual Euler characteristics of the moduli spaces of real and complex algebraic curves, Trans. A. M. S. 353 (2001), 4405-4427.

[GJ1] I.P. Goulden AND D.M. JACKSON, Maps in locally orientable surfaces and integrals over real symmetric matrices, Canad. J. Math. 49 (1997), 865-882.

[GR] I.S. Gradshteyn and I.M. Ryzhik, "Tables of Integrals, Series and Products," Academic Press, New York, 5th. ed., 1994.

[HZ] J. HARER AND D. ZAGIER, The Euler characteristic of the moduli space of curves, Invent. Math. 85 (1986), 457-485.

[M] M.L. Menta, "Random Matrices," 2nd. ed., Academic Press, 1991.

[O] A. OKоunkov, Proof of a conjecture of Goulden and Jackson, Canad. J. Math. 49 (1997), 883-886.

[St] R.P. Stanley, Some combinatorial properties of Jack symmetric functions, Adv. in Math. 77 (1989), 76-115.

[U] N. UlLAH, Ensemble average of an arbitrary number of pairs of different eigenvalues using Grassmann integration, Comm. Math. Phys. 104 (1986), 693-695. 
Appendix A. TABle of $\left[p_{2}^{m}\right] J_{N \square u}(\mathbf{x} ; \alpha)$

The following table was computed by John Stembridge, using his symmetric function package (SF) in Maple.

\begin{tabular}{|c|c|c|}
\hline$N$ & $u$ & {$\left[p_{2}^{m}\right] J_{N \square u}(\mathbf{x} ; \alpha)$} \\
\hline 1 & 2 & 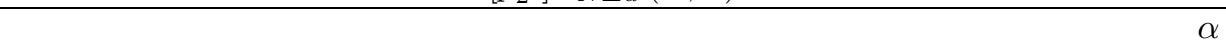 \\
\hline 1 & 4 & $3 \alpha^{2}$ \\
\hline 1 & 6 & $15 \alpha^{3}$ \\
\hline 1 & 8 & $105 \alpha^{4}$ \\
\hline 2 & 1 & -1 \\
\hline 2 & 2 & $1+\alpha+\alpha^{2}$ \\
\hline 2 & 3 & $-1-3 \alpha-5 \alpha^{2}$ \\
\hline 2 & 4 & $1+6 \alpha+17 \alpha^{2}+12 \alpha^{3}+9 \alpha^{4}$ \\
\hline 2 & 5 & $-1-10 \alpha-45 \alpha^{2}-80 \alpha^{3}-89 \alpha^{4}$ \\
\hline 2 & 6 & $1+15 \alpha+100 \alpha^{2}+315 \alpha^{3}+574 \alpha^{4}+345 \alpha^{5}+225 \alpha^{6}$ \\
\hline 2 & 7 & $-1-21 \alpha-196 \alpha^{2}-945 \alpha^{3}-2674 \alpha^{4}-3759 \alpha^{5}-3429 \alpha^{6}$ \\
\hline 3 & 2 & $5 \alpha+3 \alpha^{2}+\alpha^{3}$ \\
\hline 3 & 4 & $75 \alpha^{2}+198 \alpha^{3}+267 \alpha^{4}+108 \alpha^{5}+27 \alpha^{6}$ \\
\hline 4 & 1 & 3 \\
\hline 4 & 2 & $9+12 \alpha+17 \alpha^{2}+6 \alpha^{3}+\alpha^{4}$ \\
\hline 4 & 3 & $27+108 \alpha+267 \alpha^{2}+198 \alpha^{3}+75 \alpha^{4}$ \\
\hline 4 & 4 & $81+648 \alpha+2718 \alpha^{2}+5076 \alpha^{3}+6579 \alpha^{4}+5076 \alpha^{5}+2718 \alpha^{6}+648 \alpha^{7}+81 \alpha^{8}$ \\
\hline 5 & 2 & $89 \alpha+80 \alpha^{2}+45 \alpha^{3}+10 \alpha^{4}+\alpha^{5}$ \\
\hline 6 & 1 & -15 \\
\hline 6 & 2 & $225+345 \alpha+574 \alpha^{2}+315 \alpha^{3}+100 \alpha^{4}+15 \alpha^{5}+\alpha^{6}$ \\
\hline 7 & 2 & $3429 \alpha+3759 \alpha^{2}+2674 \alpha^{3}+945 \alpha^{4}+196 \alpha^{5}+21 \alpha^{6}$ \\
\hline 8 & 1 & $\begin{array}{lll}205 & 105\end{array}$ \\
\hline 8 & 2 & $11025+18480 \alpha+33970 \alpha^{2}+23212 \alpha^{3}+9779 \alpha^{4}+2380 \alpha^{5}+350 \alpha^{6}+28 \alpha^{7}+\alpha^{8}$ \\
\hline 9 & 1 & $1+28 \alpha+350 \alpha^{2}+2380 \alpha^{3}+9779 \alpha^{4}+23212 \alpha^{5}+33970 \alpha^{6}+18480 \alpha^{7}+11025 \alpha^{8}$ \\
\hline
\end{tabular}

Remark A.1. In each case

$$
\left[p_{2}^{m}\right] J_{N \square u}(\mathbf{x} ; 2)=(\mathbf{R H S}(\text { Theorem } 5.1) \text { or } \mathbf{R H S}(\text { Theorem } 6.1))
$$

From Proposition 2.2(b) of [St], or (17.8.5) in [M],

$$
\left[p_{2}^{t}\right] J_{1 \square u}(\mathbf{x} ; \alpha)=\alpha^{t} \frac{(2 t) !}{2^{t} t !},
$$

where $u=2 t$, in agreement for $\alpha=2$ with (6.1) specialized to $N=1$.

In addition, with $u=1,(5.6)$ gives

$$
\left[p_{2}^{n}\right] Z_{2 n \square 1}(\mathbf{x})=(-1)^{n}(2 n-1) ! !
$$


This is a result obtained by Ullah [U].

Department of Mathematics, Pennsylvania State University, University Park, PennSYLVANIA 16802 , USA

E-mail address: andrews@math.psu.edu

Department of Combinatorics and Optimization, University of Waterloo, Waterloo, Ontario, Canada

E-mail address: ipgoulde@math.uwaterloo.ca

Department of Combinatorics and Optimization, University of Waterloo, Waterloo, Ontario, Canada

E-mail address: dmjackso@math.uwaterloo.ca 